\title{
A Case of MR Imaging Detection of Hemorrhages in Cerebral Amyloid Angiopathy
}

\author{
Ruby $\mathrm{FA}^{1}$, Ahsan $\mathrm{S}^{2}$, Chandy $\mathrm{MJ}^{3}$, Alam J
}

\begin{abstract}
A 70-year-old diabetic and hypertensive male patient with mild renal impairment presented with recurrent stroke. CT scan of brain demonstrated acute hemorrhagic / nonhemorrhagic infarcts in the brain. Gradient sequences in the follow-up MRI showed extensive blooming in the cortex of both cerebral hemispheres. There were multiple foci of blooming in basal ganglia and cerebellar hemispheres. Hemosiderin staining was present in posterior fossa.

MR findings were consistent with extensive primary cerebral amyloid angiopathy (CAA).
\end{abstract}

\section{Key words}

Cerebral amyloid angiopathy (CAA), Susceptibility weighted image (SWI), T2 Gradient Echo (T2GE), subarachnoid hemorrhage (SAH), subdural hemorrhage (SAH), intraventricular hemorrhage (IVH).

\section{Introduction}

Amyloidosis is a rare systemic disease caused by extracellular deposition of beta-amyloid. Generally underlying causes of chronic renal failure, diabetes mellitus, and malignancy are present. Primary cerebral amyloid angiopathy (CAA) is a neurological condition where there is deposition of beta-amyloid in the media and adventitia of small and medium sized arteries of the brain. This can occur sporadically in absence of systemic amyloidosis. CAA accounts for $1 \%$ of stroke patient. Patient usually presents with drowsiness, headache, neurologic changes. Most of the time plain CT fails to identify the microhemorrhages occurring in the brain. CAA related ICHs are multiple, lobar and generally sparing the deep white matter, basal ganglia and brain stem.
Other manifestations are subarachnoid hemorrhage (SAH), subdural hemorrhage (SDH), intraventricular hemorrhage (IVH) and superficial hemosiderosis. ${ }^{4}$ Amyloid deposition in cerebral veins has also been described. ${ }^{1}$

\section{Case report}

A 70 year-old-man presented with recurrent stroke, vomiting for several times and excessive sweating for one week. On admission neurological examination showed GCS E4V2M5, temperature normal, blood pressure normal, planter reflex - no response on right side and equivocal on left side. Bilateral crepes were present in chest.

Patient is known diabetic and hypertensive. Biochemical tests were generally normal except serum creatinine was slightly raised. CT

1. Registrar, Department of Diagnostic and Interventional Radiology, Apollo Hospital Dhaka. 2. Consultant, Department of Diagnostic and interventional Radiology, Apollo Hospital, Dhaka. 3. Sr. Consultant, Department of Neuro surgery, Apollo Hospital, Dhaka. 4. Sr. Consultant \& Coordinator, Department of Diagnostic and Interventional Radiology, Apollo Hospital Dhaka. 
scan of brain showed acute hemorrhagic infarcts in right temporal lobe and bilateral occipital lobes, subacute right cerebellar hematoma, chronic infarcts are noted in bilateral basal ganglia, left subinsular and periventricular white matter. Chronic infarcts are also seen in left cerebellar hemisphere and pons. Since then there was progressive slowing of psychomotor activity. MRI of brain was done to exclude CAA.

MRI of brain showed multiple acute infarcts in both temporo-occipital regions. Multiple lacunar infarcts are also present in centrum semiovale and periventricular white matter. Extensive focal parenchymal hemorrhages are

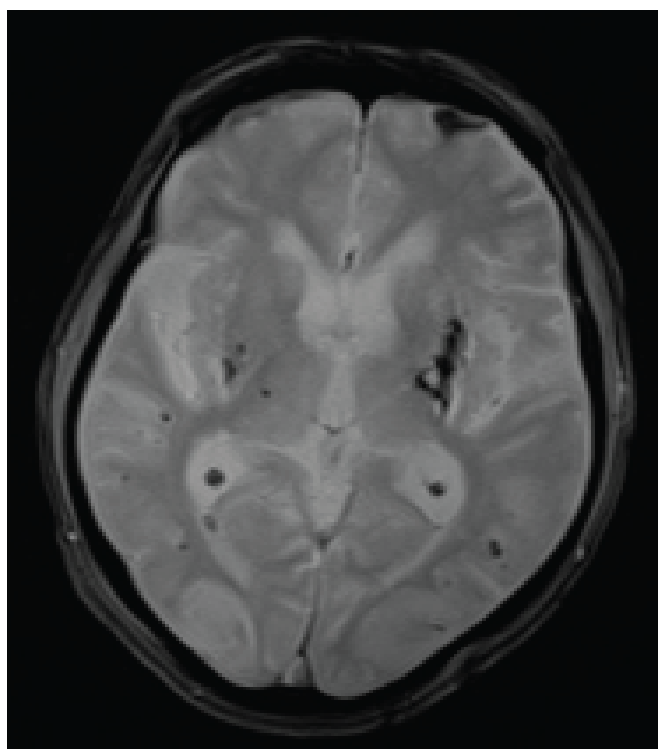

Fig.1 (A): T2*-weighted gradient-echo (T2*-GRE) Multiple acute infarcts in both temporo-occipital regions. Multiple lacunar infarcts are also present in periventricular white matter.

\section{Discussion}

Cerebral amyloid angiopathy (CAA) is a degenerative vasculopathy. It is well known to manifest as amyloid protein deposition in small arteries. CAA is now proven to be unrelated with systemic amyloidosis; it is associated with also noted in cerebellum and almost all lobes of both cerebral hemispheres. Old hemorrhagic infarcts are noted in both basal ganglia and left cerebellar hemisphere. Gradient weighted images demonstrated foci of blooming in sites of previous hemorrhages. There were few foci of cortical blooming - "black dots" in the cortex.

There was hemosiderin staining in posterior fossa. Ventricles are dilated. Periventricular hyperintensity was noted. Features were consistence with extensive CAA. During the course of illness he was evaluated for underlying bleeding diathesis and connective tissue disorder which were negative.

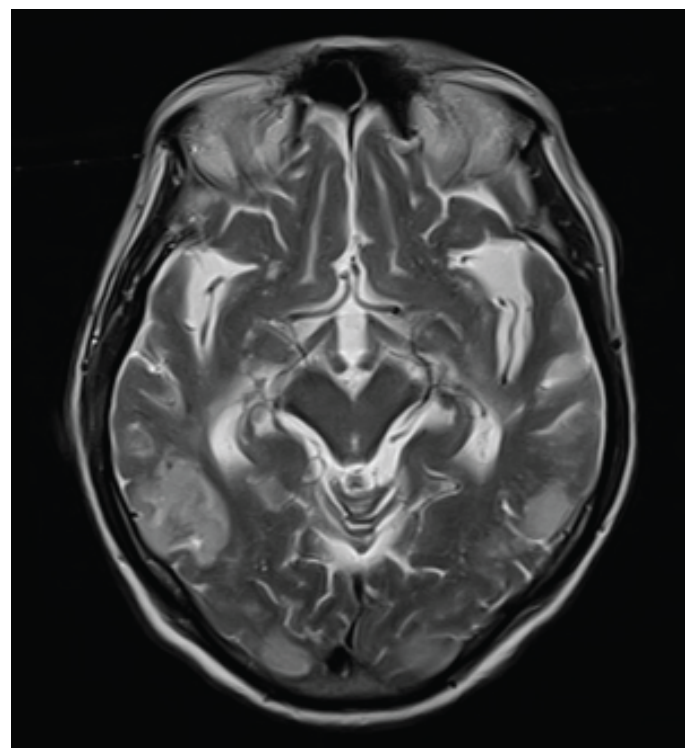

Fig.1 (B): Diffuse hyperintensity in cortex and subcortical white matter in T2WI.

increasing age, dementia, Alzheimer's disease and thus denoted as primary cerebral amyloidosis. As the beta amyloid protein builds up within the elastic lamina vessel walls, vessels lose elasticity and become fragile. Fragile vessels are more easily damaged and 
tiny hemorrhages occur in and around the arteriole vessel wall. Microbleed induced damage can cause a further loss of vessel wall or vessel wall dilatation. ${ }^{5}$ Radiological findings depends on various manifestations of disease such as:

- Microhemorrhages can not be seen in CT. T2 GE or SWI can detect the micro hemorrhages.

- Cerebral hemorrhage - appearance will vary according to age of the hemorrhage and can be see by MRI.

- Siderosis - may be seen.

- Leukoencephalopathy (inflammatory CAA) low density in $\mathrm{CT}$ and white matter hyper intensity on T2WI. ${ }^{7}$

\section{Prognosis of CAA}

Intra cranial hemorrhage - lobar intra cranial hemorrhage is associated with lower mortality rate (11-32\%) and better functional outcome.

Dementia - cognitive impairment is a common feature of CAA more than $40 \%$ of patients with $\mathrm{ICH}$ has some degree of dementia.

Vasculitis - few cases of vasculitis of different type (giant cell arthritis, rheumatoid arthritis and primary angitis of central nervous system may be reported). ${ }^{1}$

Certain patterns of lobar hemorrhage, which can be detected on $\mathrm{CT}$ and conventional MR images are suggestive of CAA. Hemorrhage involving the cortex and sub cortical white matter in frontal and parietal lobes are most common. As CAA is a vascular disease it is insensitive to diagnosis by conventional angiography.

Susceptibility weighted images (SWI) can detect more micro hemorrhage than the conventional MR angiography and digital subtraction angiogram and T2 GE technique. Micro hemorrhage contains hemosiderin which is paramagnetic relative to normal tissue and leads to large variation in local magnetic field. SWI with its sensitivity to blood products and hemorrhages is suitable to detect imaging changes consistent with CAA.

\section{Conclusion}

MRI with gradient Echo sequence is essential for diagnosis of cerebral amyloid angiopathy. This sequence is mandatory in evaluation of elderly patient (above 60).

Early detection of micro hemorrhage is important in management and monitoring of the therapeutic response of patients; especially as new therapeutic options suggest low molecular weight proteins that reduce amyloid fibril formation.

\section{Reference}

1. Menon R S , Lutsep H L. A case of cerebral amyloid angiography. 2014 Mar 27.

2. Brunot S, Fromont A, Ricolfi F, Moreau T, Giroud M. Focal subarachnoid hemorrhage and cerebral amyloid angiography: a non-fortuitous association. Rev Neurol (Paris). 2010 Jan; 166(1):83-9. doi:

10.1016/j.neurol.2009.01.037. Equp 2009 Mar 17. 3. European journal of neurology @ 2010 EFNS. Volume 18(4); p. 597-603, April 2011. Cortical subarachnoid hemorrhage in the elderly: a recurrent event probably related to cerebral amyloid angiopathy. 4. Mathew A, Anil KBP, Vivek M, Ajith S,Geeta C, Sunithi EM. Recurrent craniospinal subarachnoid hemorrhage in cerebral amyloid angiopathy. Ann Indian Acad Neurol. 2013 Jan-Mar; 16 (1):97-99. doi: 10.4103/0972-2327,107712.

5. Haackea E M, Delpropostob Z S, Chaturvedic S, Sehgalb V, Tenzere M, Neelavallid J, Kidof D. Imaging cerebral amyloid angiopathy with susceptibility -weighted imaging. AJNR Am Neuroradiol. 2007 Feb;28(2):316-7.

6. Hideya S, Akihiko U, Takayuki K, Satoshi Y, En K, Taro Y et al. Journal of Neuro inflammation. 2011; 8:116 doi:10.1186/17242-2094-8-116. 